\title{
SOME NEW ESTIMATES FOR DISTANCES IN ANALYTIC FUNCTION SPACES OF SEVERAL COMPLEX VARIABLES AND DOUBLE BERGMAN REPRESENTATION FORMULA
}

\author{
ROMI SHAMOYAN ${ }^{1}$ AND MEHDI RADNIA ${ }^{2}$
}

\begin{abstract}
Using double Bergman representation formula we provide new sharp estimates for distances from fixed analytic functions to some subspaces of holomorphic functions in unit polydisk and unit ball. We will enlarge the list of previously known assertions of this type obtained recently by $\mathrm{R}$. Zhao and $\mathrm{W}$. Xu.
\end{abstract}

\section{INTRODUCTION AND NOTATIONS}

Let $\mathbb{D}$ be, as usual, the unit disk on the complex plane, $d m_{2}(z)$ the normalized Lebesgue measure on $\mathbb{D}$ so that $m_{2}(\mathbb{D})=1$ and $d \xi$ be the Lebesgue measure on the circle $T=\{\xi$ : $|\xi|=1\}$. Let further $H(\mathbb{D})$ be the space of all holomorphic functions on the unit disk $\mathbb{D}$.

For $f \in H(\mathbb{D})$ and $f(z)=\sum_{k} a_{k} z^{k}$, we define the fractional derivative of the function $f$ as usual in the following manner

$$
D^{\alpha} f(z)=\sum_{k=0}^{\infty}(k+1)^{\alpha} a_{k} z^{k}, \alpha \in \mathbb{R} .
$$

We will write $D f(z)$ if $\alpha=1$. Obviously, for all $\alpha \in R, D^{\alpha} f \in H(\mathbb{D})$.

For $a \in \mathbb{D}$, let $g(z, a)=\log \frac{1}{\left|\phi_{a}(z)\right|}$ be the Green's function for $\mathbb{D}$ with pole at $a$, where $\phi_{a}(z)=\frac{a-z}{1-\bar{a} z}$. For $0<p<\infty,-2<q<\infty, 0<s<\infty,-1<q+s<\infty$, we say that

Date: Received: 07 Nov. 2009.

* Corresponding author.

2000 Mathematics Subject Classification. Primary 30D45.

Key words and phrases. holomorphic function, distance function, Bloch-type space, Bergman-type classes, unit ball, unit polydisk. 
$f \in F(p, q, s)$, if $f \in H(\mathbb{D})$ and

$$
\|F\|_{F(p, q, s)}^{p}=\sup _{a \in \mathbb{D}} \int_{\mathbb{D}}|D f(z)|^{p}\left(1-|z|^{2}\right)^{q} g^{s}(z, a) d m_{2}(z)<\infty .
$$

As we know [14], if $0<p<\infty,-2<q<\infty, 0<s<\infty,-1<q+s<\infty, f \in F(p, q, s)$, if and only if

$$
\int_{\mathbb{D}}|D f(z)|^{p}\left(1-|z|^{2}\right)^{q}\left(1-\left|\phi_{a}(z)\right|\right)^{s} d m_{2}(z)<\infty .
$$

It is known also that $F(2,0,1)=B M O A$.

We recall that weighted Bloch class $\mathbf{B}^{\alpha}(\mathbb{D}), \alpha>0$, is the collection of the analytic functions on the unit disk satisfying

$$
\|f\|_{\mathbf{B}^{\alpha}}=\sup _{z \in \mathbb{D}}|D f(z)|\left(1-|z|^{2}\right)^{\alpha}<\infty .
$$

$\mathbf{B}^{\alpha}(\mathbb{D})$ is a Banach space with the norm $\|f\|_{\mathbf{B}^{\alpha}}$. Note $\mathbf{B}^{1}(\mathbb{D})=B(D)$ is a classical Bloch class(see [2] and the references there).

The well-known so called "duality" approach to extremal problems in theory of analytic functions leads to the following general formula

$$
\operatorname{dist}_{Y}(g, X)=\sup _{l \in X^{\perp},\|l\| \leq 1}|l(g)|=\inf _{\varphi \in X}\|g-\varphi\|_{Y},
$$

where $g \in Y, X$ is subspace of a normed space $Y, Y \in H(D)$ and $X^{\perp}$ it is ortogonal complement in $Y^{*}$, the dual space of $Y$ and $l$ is a linear functional on $Y$ (see [6]). Various extremal problems in Hp Hardy classes in $\mathbb{D}$ based on duality approach we mentioned were discussed in [3, Chapter 8]. In particular for a function $K \in L^{q}(T)$ the following equality holds (see [3]), $1<p<\infty, \frac{1}{p}+\frac{1}{q}=1$,

$$
\operatorname{dist}_{L^{q}}\left(K, H^{q}\right)=\inf _{g \in H^{q}, K \in L^{q}}\|K-g\|_{H^{q}}=\sup _{f \in H^{p},\|f\|_{H^{p}} \leq 1} \frac{1}{2 \pi}\left|\int_{|\xi|=1} f(\xi) K(\xi) d \xi\right| .
$$

It is well known if $p>1$ then inf-dual extremal problem in analytic $H^{p}$ Hardy classes has a solution, it is unique if an extremal function exists (see [3]). Note also that extremal problems for $H^{p}$ spaces in multiply connected domains were studied before in [1] , [7] and [8]. Various new results on extremal problems in $A^{p}$ Bergman class and it is subspaces were obtained recently by many authors (see [5] and the references there). In this paper, we will provide direct proofs for estimation of

$$
\operatorname{dist}_{Y}(f, X)=\inf _{g \in X}\|f-g\|_{Y}, X \subset Y, f \in Y, X, Y \subset H(\mathbb{D})
$$

in higher dimension.

Let further $\Omega_{\alpha, \varepsilon}^{k}=\left\{z \in \mathbb{D}:\left|D^{k} f(z)\right|\left(1-|z|^{2}\right)^{\alpha} \geq \varepsilon\right\}, \Omega_{\alpha, \varepsilon}^{0}=\Omega_{\alpha, \varepsilon}$.

Applying famous Fefferman duality theorem, P. Jones proved the following.

Theorem A.([4], [14]) Let $f \in B$. Then the following are equivalent:

(1) $d_{1}=\operatorname{dist}_{\mathbf{B}}(f, B M O A)$;

(2) $d_{2}=\inf \left\{\varepsilon: \chi_{\Omega_{1, \varepsilon}^{1}}(z) \frac{d m_{2}(z)}{1-|z|^{2}}\right.$ is a Carleson measure $\}$, 
where $\chi$ denotes characteristic function of the mentioned set.

Recently, R. Zhao (see [14]) and W. Xu (see [13]) obtained results on distances from Bloch functions to some Möbius invariant function spaces in a relatively direct way. The goal of this paper is to develop further their ideas and present new theorems in analytic function spaces in higher dimension.

In next sections various assertions for distance function will be given. We will indicate proofs of some assertions in details, short sketches in some cases will be also provided.

Throughout the paper, we write $C$ ( sometimes with indexes) to denote a positive constant which might be different at each occurrence (even in a chain of inequalities) but is independent of the functions or variables being discussed.

We will write for two expressions $A \lesssim B$ if there is a positive constant $\mathrm{C}$ such that $A<C B$.

\section{MAin Results}

In this section applying rather unusual "double" Bergman representation formula, we indicate a precise formula for "dist" function that allows to estimate distances from any function from certain analytic BMOA type class to certain weighted Bergman space. The simple nature of our proof allows to consider more general weighted Bergman classes, but we restrict ourselves to standart weights. We assume in advance that inf of any set below and the set itself can not be empty. We denote below everywhere by $\mathbb{D}^{n}$ as usual the unit polydisk (see for example [2] and references there) and use below all standart notations of function theory in polydisk that can be found for example in [2].

Let $\alpha>r, r>1, \alpha>1$. Then obviously

$$
\begin{aligned}
\|f\|_{Q_{r, \alpha}}= & \sup _{a_{j} \in \mathbb{D}} \int_{\mathbb{D}} \ldots \int_{\mathbb{D}} \frac{\left|f\left(z_{1}, \ldots, z_{n}\right)\right| \times \prod_{k=1}^{n}\left(1-\left|z_{k}\right|\right)^{\alpha-2}}{\prod_{k=1}^{n}\left(\left|1-\overline{a_{k}} z_{k}\right|\right)^{\alpha}} d m_{2 n}(z) \times \prod_{k=1}^{n}\left(1-\left|a_{k}\right|\right)^{r} \\
& \leq C\|f\|_{A_{r-2}^{1}}=C \int_{\mathbb{D}^{n}}\left|f\left(z_{1}, \ldots, z_{n}\right)\right| \times \prod_{k=1}^{n}\left(1-\left|z_{k}\right|\right)^{r-2} d m_{2 n}(z)
\end{aligned}
$$

where $\mathbb{D}^{n}$ is a unit polydisk, $f \in H\left(\mathbb{D}^{n}\right), H\left(\mathbb{D}^{n}\right)$ is a space of holomorphic functions in $\mathbb{D}^{n}$ and $d m_{2 n}(z)$ is a Lebesque measure on $\mathbb{D}^{n}$. So we have a natural exremal problem in polydisk to find

$$
\operatorname{dist}_{Q_{r, \alpha}}\left(f, A_{r-2}^{1}\right)=\inf _{g \in A_{r-2}^{1}}\|f-g\|_{Q_{r, \alpha}}, f \in Q_{r, \alpha} .
$$

For $f \in H\left(\mathbb{D}^{n}\right)$, we define a set

$$
\begin{aligned}
& \quad N_{f}=N_{f, \varepsilon}^{\alpha, r}=\left\{a \in \mathbb{D}^{n}: \int_{\mathbb{D}^{n}} \frac{\left|f\left(z_{1}, \ldots, z_{n}\right)\right| \prod_{k=1}^{n}\left(1-\left|z_{k}\right|\right)^{\alpha-2}}{\prod_{k=1}^{n}\left(\left|1-\overline{a_{k}} z_{k}\right|\right)^{\alpha}} d m_{2 n}(z) \times \prod_{k=1}^{n}\left(1-\left|a_{k}\right|\right)^{r} \geq \varepsilon\right\}, \\
& \varepsilon>0, \alpha>1, r>0 .
\end{aligned}
$$

Remark. Note $A_{r-2}^{1}$ is a standard Bergman class in polydisk $\mathbb{D}^{n}$ studied before by various authors (see for example [2]). Classes $Q_{r, \alpha}$ in disk $\mathbb{D}$ are so called BMOA type spaces were also under investigation by many authors recently (see [11], [12]). 
Appling classical Bergman representation for the unit disk $n$ times by each variable we get Bergman representation formula in polydisk.

$$
f\left(z_{1}, \ldots, z_{n}\right)=C_{\gamma} \int_{\mathbb{D}^{n}} \frac{f\left(w_{1}, \ldots, w_{n}\right) \prod_{k=1}^{n}\left(1-\left|w_{k}\right|\right)^{\gamma}}{\prod_{k=1}^{n}\left(1-\overline{w_{k}} z_{k}\right)^{\gamma+2}} d m_{2 n}(w)
$$

where $C_{\gamma}$ is constant, $z_{j} \in \mathbb{D}, j=1, \ldots, n, \gamma>-1, f \in H\left(\mathbb{D}^{n}\right)$. Choose $\gamma_{1}>-1$, then applying Bergman representation formula twice we have a double Bergman representation for analytic $f$ function in polydisk

$$
\begin{gathered}
f\left(z_{1}, \ldots, z_{n}\right)=C_{\gamma} C_{\gamma_{1}} \int_{\mathbb{D}^{n}} \frac{\prod_{k=1}^{n}\left(1-\left|w_{k}\right|\right)^{\gamma}}{\prod_{k=1}^{n}\left(1-\overline{w_{k}} z_{k}\right)^{\gamma+2}} \times \\
\int_{\mathbb{D}^{n}} \frac{\prod_{k=1}^{n}\left(1-\left|\widetilde{w_{k}}\right|\right)^{\gamma_{1}} f\left(\widetilde{w_{1}}, \ldots, \widetilde{w_{n}}\right)}{\prod_{k=1}^{n}\left(1-\widetilde{w_{k}} w_{k}\right)^{\gamma_{1}+2}} d m_{2 n}(\widetilde{w}) d m_{2 n}(w)=f_{1}\left(z_{1}, \ldots, z_{n}\right)+f_{2}\left(z_{1}, \ldots, z_{n}\right),
\end{gathered}
$$

and

$$
\begin{gathered}
f_{1}\left(z_{1}, \ldots, z_{n}\right)=C \int_{\mathbb{D}^{n}} \int_{\mathbb{D}^{n} \backslash N_{f}} \frac{\prod_{k=1}^{n}\left(1-\left|w_{k}\right|\right)^{\gamma}}{\prod_{k=1}^{n}\left(1-\overline{w_{k}} z_{k}\right)^{\gamma+2}} \times \\
\frac{\prod_{k=1}^{n}\left(1-\left|\widetilde{w_{k}}\right|\right)^{\gamma_{1}} f\left(\widetilde{w_{1}}, \ldots, \widetilde{w_{n}}\right)}{\prod_{k=1}^{n}\left(1-\widetilde{\widetilde{w_{k}}} w_{k}\right)^{\gamma_{1}+2}} d m_{2 n}(\widetilde{w}) d m_{2 n}(w), \\
f_{2}\left(z_{1}, \ldots, z_{n}\right)=C \int_{\mathbb{D}^{n}} \int_{N_{f}} \frac{\prod_{k=1}^{n}\left(1-\left|w_{k}\right|\right)^{\gamma}}{\prod_{k=1}^{n}\left(1-\overline{w_{k}} z_{k}\right)^{\gamma+2}} \times \\
\frac{\prod_{k=1}^{n}\left(1-\left|\widetilde{w_{k}}\right|\right)^{\gamma_{1}} f\left(\widetilde{w_{1}}, \ldots, \widetilde{w_{n}}\right)}{\prod_{k=1}^{n}\left(1-\widetilde{\widetilde{w_{k}}} w_{k}\right)^{\gamma_{1}+2}} d m_{2 n}(\widetilde{w}) d m_{2 n}(w),
\end{gathered}
$$

where $d m_{2 n}(w)=d m_{2}\left(w_{1}\right) \ldots d m_{2}\left(w_{n}\right), C=C_{\gamma, \gamma_{1}}$.

Fix some $\varepsilon>0$. Our task to show $f_{1} \in Q_{r, \alpha},\left\|f_{1}\right\|_{Q_{r, \alpha}} \leq C \varepsilon, f_{2} \in A_{r-2}^{1}$. Then we will have

$$
\operatorname{dist}_{Q_{r, \alpha}}\left(f, A_{r-2}^{1}\right) \leq C\left\|f-f_{2}\right\|_{Q_{r, \alpha}}=C\left\|f_{1}\right\|_{Q_{r, \alpha}} \leq C \varepsilon, f \in Q_{r, \alpha} \text {. }
$$

Moreover it turns out the reverse estimate is also true and we have the following sharp theorem. We assume also that all inf everywhere in paper are taken from not empty sets.

Theorem 2.1. Let $r\left\langle\alpha-1, r>1, \alpha>1, f \in Q_{r, \alpha}\right.$, then the following are equivalent:

(1) $\left[\right.$ dist $\left._{Q_{r, \alpha}}\left(f, A_{r-2}^{1}\right)\right]$;

(2) $\inf \left\{\varepsilon>0, \int_{\mathbb{D}^{n}}\left(\chi_{N_{f}}\right)\left(a_{1}, \ldots, a_{n}\right) \prod_{k=1}^{n}\left(1-\left|a_{k}\right|\right)^{-2} d m_{2 n}(a)<\infty\right\}$, where $\chi_{M}$ is characteristic function of a set $M, M \subset \mathbb{D}^{n}$;

Proof. Let us show first the implication $(2) \rightarrow(1)$ using arguments we provided above. We have for $\gamma>r-2$

$$
\begin{gathered}
\left\|f_{2}\right\|_{A_{r-2}^{1}}=\int_{\mathbb{D}^{n}} \prod_{k=1}^{n}\left(1-\left|z_{k}\right|\right)^{r-2}\left|f_{2}\left(z_{1}, \ldots, z_{n}\right)\right| d m_{2 n}(z) \leq \\
C \int_{\mathbb{D}^{n}} \prod_{k=1}^{n}\left(1-\left|z_{k}\right|\right)^{r-2} \int_{\mathbb{D}^{n}} \int_{N_{f}} \frac{\prod_{k=1}^{n}\left(1-\left|w_{k}\right|\right)^{\gamma}}{\left|\prod_{k=1}^{n}\left(1-w_{k} z_{k}\right)^{\gamma+2}\right|} \times
\end{gathered}
$$




$$
\int_{\mathbb{D}^{n}} \frac{\prod_{k=1}^{n}\left(1-\left|\widetilde{w_{k}}\right|\right)^{\gamma_{1}}\left|f\left(\widetilde{w_{1}}, \ldots, \widetilde{w_{n}}\right)\right|}{\left|\prod_{k=1}^{n}\left(1-\widetilde{w_{k}} w_{k}\right)^{\gamma_{1}+2}\right|} d m_{2 n}(\widetilde{w}) d m_{2 n}(w) d m_{2 n}(z),
$$

Hence using Fubinis theorem and putting $\gamma_{1}=\alpha-2$, we will have

$$
\begin{gathered}
\left\|f_{2}\right\|_{A_{r-2}^{1}}=C \int_{\mathbb{D}^{n}} \int_{N_{f}} \frac{\left|f\left(\widetilde{w_{1}}, \ldots, \widetilde{w_{n}}\right)\right| \prod_{k=1}^{n}\left(1-\left|\widetilde{w_{k}}\right|\right)^{\gamma_{1}}}{\left|\prod_{k=1}^{n}\left(1-\widetilde{w_{k}} w_{k}\right)^{\gamma_{1}+2}\right|} \times \prod_{k=1}^{n}\left(1-\left|w_{k}\right|\right)^{\gamma} \\
\times \prod_{k=1}^{n}\left(1-\left|w_{k}\right|\right)^{r-\gamma-2} d m_{2 n}(\widetilde{w}) d m_{2 n}(w) \leq \sup _{w_{k}} C \int_{\mathbb{D}^{n}} \frac{\mid f\left(\widetilde{w_{1}}, \ldots, \widetilde{w_{n}}\right) \prod_{k=1}^{n}\left(1-\left|\widetilde{w_{k}}\right|\right)^{\alpha-2}}{\prod_{k=1}^{n}\left(\left|1-\widetilde{w_{k}} \overline{w_{k}}\right|\right)^{\alpha}} \\
\prod_{k=1}^{n}\left(1-\left|w_{k}\right|\right)^{r}\left(\int_{\mathbb{D}^{n}}\left(\chi_{N_{f}}\right)\left(w_{1}, \ldots, w_{n}\right) \prod_{k=1}^{n}\left(1-\left|w_{k}\right|\right)^{-2} d m_{2 n}(w)\right)
\end{gathered}
$$

We used that

$$
\int_{\mathbb{D}^{n}} \frac{\prod_{k=1}^{n}\left(1-\left|w_{k}\right|\right)^{t_{1}} d m_{2 n}(w)}{\prod_{k=1}^{n}\left|\left(1-\widetilde{w_{k}} z_{k}\right)\right|^{t_{2}}} \asymp C \prod_{k=1}^{n}\left(1-\left|z_{k}\right|\right)^{t_{1}+2-t_{2}}, t_{1}>-1, t_{2}>t_{1}+2, z \in \mathbb{D}^{n} .
$$

(See [2]). Now we show $\left\|f_{1}\right\|_{Q_{r, \alpha}} \leq C \varepsilon$. We have using (3) and Fubinis theorem for $\gamma>r-1$

$$
\begin{gathered}
\left\|f_{1}\right\|_{Q_{r, \alpha}}=\left(\sup _{a_{j} \in \mathbb{D}}\right) \int_{\mathbb{D}^{n}} \frac{\left|f_{1}\left(z_{1}, \ldots, z_{n}\right)\right| \prod_{k=1}^{n}\left(1-\left|z_{k}\right|\right)^{\alpha-2}}{\prod_{k=1}^{n}\left|\left(1-\overline{a_{k}} z_{k}\right)\right|^{\alpha}} d m_{2 n}(z) \times \prod_{k=1}^{n}\left(1-\left|a_{k}\right|\right)^{r} \\
\leq\left(C \sup _{a_{j} \in \mathbb{D}}\right) \int_{\mathbb{D}^{n}} \int_{\mathbb{D}^{n}} \int_{\mathbb{D}^{n} \backslash N_{f}} \frac{\prod_{k=1}^{n}\left(1-\left|w_{k}\right|\right)^{\gamma} \prod_{k=1}^{n}\left(1-\left|z_{k}\right|\right)^{\alpha-2}}{\prod_{k=1}^{n}\left|\left(1-\overline{w_{k}} z_{k}\right)\right|^{\gamma+2}} \times \prod_{k=1}^{n}\left(1-\left|a_{k}\right|\right)^{r} \\
\times \frac{\prod_{k=1}^{n}\left(1-\left|\widetilde{w_{k}}\right|\right)^{\alpha-2}\left|f\left(\widetilde{w_{1}}, \ldots, \widetilde{w_{n}}\right)\right|}{\prod_{k=1}^{n}\left|\left(1-\widetilde{w_{k}} w_{k}\right)^{\alpha}\right|} d m_{2 n}(z) d m_{2 n}(\widetilde{w}) d m_{2 n}(w) \leq \\
(C \varepsilon) \sup _{a_{j} \in \mathbb{D}} \int_{\mathbb{D}^{n}} \frac{\prod_{k=1}^{n}\left(1-\left|a_{k}\right|\right)^{r} \prod_{k=1}^{n}\left(1-\left|w_{k}\right|\right)^{\alpha-2-r}}{\prod_{k=1}^{n}\left|\left(1-\overline{a_{k}} w_{k}\right)\right|^{\alpha}} d m_{2 n}(w) \leq C_{1} \varepsilon .
\end{gathered}
$$

So we showed one part of our theorem. To show the reverse we assume the reverse to that assertion is true. Hence by assumption there are $\varepsilon, \varepsilon_{1}, \varepsilon>0, \varepsilon_{1}>0$ and there is $f_{\varepsilon_{1}} \in A_{r-2}^{1}$, $\varepsilon>\varepsilon_{1},\left\|f-f_{\varepsilon_{1}}\right\|_{Q_{r, \alpha}} \leq \varepsilon_{1}$ and $K=\int_{\mathbb{D}^{n}}\left(\chi_{N_{f}}(a)\right)\left(a_{1}, \ldots, a_{n}\right) \prod_{k=1}^{n}\left(1-\left|a_{k}\right|\right)^{-2} d m_{2 n}(a)=\infty$. Using this we arrive easily to contradiction. Indeed we have $\widetilde{f}=f-f_{\varepsilon_{1}}, \tau>\rho, \beta-\tau+\rho=$ $r-2, \rho>1, \beta>-1, \tau>0, a \in \mathbb{D}^{n}$

$$
\begin{gathered}
\mathcal{M}(a)=\int_{\mathbb{D}^{n}} \frac{\left|f_{\varepsilon_{1}}\left(z_{1}, \ldots, z_{n}\right)\right| \prod_{k=1}^{n}\left(1-\left|z_{k}\right|\right)^{\beta}}{\prod_{k=1}^{n}\left(\left|1-\overline{a_{k}} z_{k}\right|\right)^{\tau}} d m_{2 n}(z) \prod_{k=1}^{n}\left(1-\left|a_{k}\right|\right)^{\rho} \geq \\
\prod_{k=1}^{n}\left(1-\left|a_{k}\right|\right)^{\rho} \int_{\mathbb{D}^{n}} \frac{\left|f\left(z_{1}, \ldots, z_{n}\right)\right| \prod_{k=1}^{n}\left(1-\left|z_{k}\right|\right)^{\beta}}{\left|\prod_{k=1}^{n}\left(1-\overline{a_{k}} z_{k}\right)\right|^{\tau}} d m_{2 n}(z) \\
-\left(\sup _{a \in \mathbb{D}^{n}}\right) \int_{\mathbb{D}^{n}} \frac{\left|f_{\varepsilon_{1}}(z)-f(z)\right| \prod_{k=1}^{n}\left(1-\left|z_{k}\right|\right)^{\beta}}{\left|\prod_{k=1}^{n}\left(1-\overline{a_{k}} z_{k}\right)\right|^{\tau}} d m_{2 n}(z) \prod_{k=1}^{n}\left(1-\left|a_{k}\right|\right)^{\rho}
\end{gathered}
$$


Hence

$$
\mathcal{M}(a) \geq\left(\left(\varepsilon-\varepsilon_{1}\right)\left(\chi_{N_{f}}(a)\right)\right.
$$

and by (3) after choosing appropriate $\rho>1$ and $\beta>-1$, we will have

$$
\int_{\mathbb{D}^{n}}\left|f_{\varepsilon_{1}}(z)\right|\left(1-\left|z_{1}\right|\right)^{r-2}\left(1-\left|z_{n}\right|\right)^{r-2} d m_{2 n}(z) \geq\left(\varepsilon-\varepsilon_{1}\right) K
$$

So we have an obvious contradiction. Our theorem 2.1 is proved.

Let us note that similar results holds with similar proofs in unit ball $B$ of $\mathbb{C}^{n}$ and classes on subframes $\widetilde{\mathbb{D}}^{n}$ and expanded disk $\mathbb{D}_{*}^{n}$.

$$
\begin{aligned}
\mathbb{D}_{*}^{n} & =\left\{z \in \mathbb{D}^{n}, z=\left(z_{1}, \ldots, z_{n}\right), z_{j}=\left|z_{j}\right| \xi,\left|z_{j}\right| \in(0,1), \xi \in T=\{|z|=1\} .\right. \\
\widetilde{\mathbb{D}}^{n} & =\left\{z \in \mathbb{D}^{n}, z=\left(z_{1}, \ldots, z_{n}\right), z_{j}=|z| \xi_{j}, \xi_{j} \in T, j=1, \ldots, n,|z| \in(0,1)\right\} .
\end{aligned}
$$

Namely we consider Bergman classes $A_{\alpha}^{1}\left(\mathbb{D}_{*}^{n}\right)$ and $A_{\alpha}^{1}\left(\widetilde{\mathbb{D}}^{n}\right)$ defined us

$$
\begin{gathered}
A_{\alpha}^{1}\left(\mathbb{D}_{*}^{n}\right)=\left\{f \in H\left(\mathbb{D}^{n}\right): \int_{0}^{1} \int_{0}^{1} \int_{T}\left|f\left(r_{1} \xi, \ldots, r_{n} \xi\right)\right| \prod_{k=1}^{n}\left(1-r_{k}\right)^{\alpha} d r_{1} \ldots d r_{n} d \xi<\infty\right\} . \\
A_{\alpha}^{1}\left(\widetilde{\mathbb{D}}^{n}\right)=\left\{f \in H\left(\mathbb{D}^{n}\right): \int_{T^{n}} \int_{0}^{1}\left|f\left(r \xi_{1}, \ldots, r \xi_{n}\right)\right|(1-r)^{\alpha} d r d m_{n}(\xi)<\infty\right\} .
\end{gathered}
$$

Where $d m_{n}(\xi)$ is standard Lebesque measure in $T^{n}, \alpha>-1$ and $H\left(\mathbb{D}^{n}\right)$ as above is a space of holomorphic function in $\mathbb{D}^{n}$ and $Q_{r, \alpha}\left(\mathbb{D}_{*}^{n}\right)$ and $Q_{r, \alpha}\left(\widetilde{\mathbb{D}}^{n}\right)$ are defined by replacing polydisk $\mathbb{D}^{n}$ by $\mathbb{D}_{*}^{n}$ or $\widetilde{\mathbb{D}}^{n}$ accordingly Note analytic classes on subframes and expanded disk were studied before in [9], [10]. We also note that proofs of mentioned assertions can be obtained by small modification of proofs of our main theorem 2.1 .

Let $\widetilde{m}_{2 n}(w)$ and $m^{*}{ }_{2 n}(w)$ be Lebesque measure in $\widetilde{\mathbb{D}}^{n}$ and $\mathbb{D}_{*}^{n}$, obviously for $\alpha>\frac{1}{n}, r>\frac{1}{n}$, $\alpha>r$

$$
\begin{gathered}
\|f\|_{Q_{r, \alpha}^{1}\left(\widetilde{\mathbb{D}}^{n}\right)}=\left(\sup _{a_{j} \in \mathbb{D}}\right) \int_{0}^{1} \int_{T^{n}} \frac{|f(w)|(1-|w|)^{n \alpha-2} d \widetilde{m}_{2 n}(w)}{\prod_{k=1}^{n}\left(\left|1-\overline{a_{k}} w_{k}\right|\right)^{\alpha}} \times \prod_{k=1}^{n}\left(1-\left|a_{k}\right|\right)^{r} \leq \\
C \int_{0}^{1} \int_{T^{n}}|f(w)|(1-|w|)^{n \alpha-2-(\alpha-r) n} d \widetilde{m}_{2 n}(w) \leq C \int_{0}^{1} \int_{T^{n}}|f(w)|(1-|w|)^{r n-2} d \widetilde{m}_{2 n}(w) . \\
\|f\|_{Q_{r, \alpha}^{2}\left(\mathbb{D}_{*}^{n}\right)}=\left(\sup _{a_{j} \in \mathbb{D}}\right) \int_{0}^{1} \ldots \int_{0}^{1} \int_{T} \frac{|f(w)| \prod_{k=1}^{n}\left(1-\left|w_{k}\right|\right)^{\alpha-2}}{\prod_{k=1}^{n}\left(\left|1-\overline{a_{k}} w_{k}\right|\right)^{\alpha}} d m_{2 n}^{*}(w)\left(\prod_{k=1}^{n}\left(1-\left|a_{k}\right|\right)^{r}\right) \\
\leq C\left(\|f\|_{A_{r-2}^{1}}\right)=C \int_{0}^{1} \ldots \int_{0}^{1} \int_{T}|f(w)|\left(1-\left|w_{1}\right|\right)^{r-2} \ldots\left(1-\left|w_{n}\right|\right)^{r-2} d m^{*}{ }_{2 n}(w),
\end{gathered}
$$

$\alpha>r, r>1, \alpha>1$.

So the problem of finding estimate for $\left(\operatorname{dist}_{Q_{r, \alpha}^{2}}\left(f, A_{r-2}^{1}\left(\mathbb{D}_{*}^{n}\right)\right), f \in\left(Q_{r, \alpha}^{2}\right)\left(\mathbb{D}_{*}^{n}\right)\right.$; or $\left(\operatorname{dist}_{Q_{r, \alpha}^{1}}\left(f, A_{r n-2}^{1}\left(\widetilde{\mathbb{D}}^{n}\right)\right)\right.$, $f \in\left(Q_{r, \alpha}^{1}\right)\left(\widetilde{\mathbb{D}}^{n}\right)$; appears naturally in subframe $\widetilde{\mathbb{D}}^{n}$ and expanded disk $\mathbb{D}_{*}^{n}$ also.

We formulate a version of theorem 2.1 for unit ball, we use standard notations from [15]. 
Let $d v(z)$ volume measure on ball $\mathbb{B}, d \sigma(\xi)$ standard Lebesque measure on $\mathbb{S}$ sphere. We have for $r>n, \alpha>r, \alpha>n$

Let

$$
\begin{aligned}
\|f\|_{Q_{r, \alpha}(\mathbb{B})} & =\left(\sup _{a \in \mathbb{B}}\right) \int_{\mathbb{B}} \frac{|f(w)| \prod_{k=1}^{n}(1-|w|)^{\alpha-n-1}}{(|1-<w, a>|)^{\alpha}} d v(z) \times(1-|a|)^{r} \leq \\
& C \int_{\mathbb{B}}|f(w)|(1-|w|)^{r-n-1} d v(w)=\|f\|_{A_{r-n-1}^{1}(\mathbb{B})}
\end{aligned}
$$

$$
\widetilde{N}_{f}=\left\{a \in \mathbb{B}:\left(\int_{\mathbb{B}} \frac{|f(w)|(1-|w|)^{\alpha-n-1} d v(w)}{(|1-<a, w>|)^{\alpha}}\right)(1-|a|)^{r} \geq \varepsilon\right\} .
$$

Theorem 2.2. Let $r>n, \alpha>n, r<\alpha-n, f \in Q_{r, \alpha}(\mathbb{B})$, then the following are equivalent:

(1) $\left[\operatorname{dist}_{Q_{r, \alpha}(\mathbb{B})}\left(f, A_{r-n-1}^{1}(\mathbb{B})\right)\right]$;

(2) $\left.\inf \left\{\varepsilon>0, \int_{\mathbb{B}}\left[\chi_{\widetilde{N}_{f}}\right)(a)\right](1-|a|)^{-n-1} d \sigma(a)<\infty\right\}$;

Acknowledgements. We would like to thank Professor Reza Saadati for his valuable suggestions during the preparation of this paper to publication and his encouragement.

\section{REFERENCES}

[1] L. V. Ahlfors, Bounded analytic functions, Duke Math Journal, 14 (1947), 1-14.

[2] M. M. Djrbashian, F. A. Shamoian, Topics in theory of $A_{\alpha}^{p}$ classes, Teubner zur Mathematics, 1988, v105.

[3] P. L. Duren, Theory of $H^{p}$ spaces, Academic Press, New York, 1970.

[4] P. G. Ghatage, D. Zheng, Analytic functions of bounded mean oscillation and the Bloch space, Integr. Equat. Oper. Theory, 17 (1993), 501-515.

[5] D. Khavinson, M. Stessin, Certain linear extremal problems in Bergman spaces of analytic function, Indiana Univ. Math. J., 3(46) (1997), 933-974.

[6] S. Ya Khavinson, On an extremal problem in the theory of analytic function, Russian Math. Surv., 4(1949), 158-159.

[7] W. Rudin, Analytic functions of class $H^{p}$, Trans AMS, 78 (1955), 46-66.

[8] W. Rudin, Function theory in polydisks, Benjamin, New York, 1969.

[9] R. Shamoyan, O. Mihic, Analytic classes on subframe and expanded disk and the $R^{s}$ differential operator in polydisk, Journal of Inequalities and applications, 2010.

[10] R. Shamoyan, S. Li, On some properties of a differential operator on the polydisk, Banach Journal of Mathematical Analysis, 3(1) (2009), 68-84.

[11] J. Xiao, Holomorphic $Q_{p}$ classes, Lecture Notes in Math,1767, Springer-Verlag, 2006.

[12] J. Xiao, Geometric $Q_{p}$ functions, Frontiers in math, Birkhauser-Verlag, 2006.

[13] W. Xu, Distances from Bloch functions to some Möbius invariant function spaces in the unit ball of $C^{n}$, J. Funct. Spaces Appl., 7(1) (2009), 91-104.

[14] R. Zhao, Distances from Bloch functions to some Möbius invariant function spaces, Ann. Acad. Sci. Fenn. Math., 33 (2008), 303-313.

[15] K. Zhu, Spaces of Holomorphic Functions in the Unit Ball, Graduate Texts in Mathematics, 226, Springer-Verlag, New York, 2005.

1 Department of Mathematics, Bryansk State University, Bryansk, Russia.

E-mail address: rshamoyan@yahoo.com

2 Department of Mathematics, Tabriz University, Tabriz, Iran.

E-mail address: mehdi.radnia@gmail.com 